\title{
PERIODS OF PERIODIC SOLUTIONS AND THE LIPSCHITZ CONSTANT ${ }^{1}$
}

\author{
JAMES A. YORKE
}

Let $x(t)$ be a nonconstant periodic solution with period $p$ of

$$
x^{\prime}=F(x) \quad\left({ }^{\prime}=d / d t\right)
$$

where $F: \Omega \rightarrow R^{n}$ for some set $\Omega \subset R^{n}$. Let $\|\cdot\|$ denote the Euclidean norm. We say $F$ has (Euclidean) Lipschitz constant $L$ if

$$
\left\|F\left(x_{1}\right)-F\left(x_{2}\right)\right\| \leqq L\left\|x_{1}-x_{2}\right\| \quad \text { for each } x_{1}, x_{2} \in \Omega \text {. }
$$

There is a simple relationship between $L$ and $p$.

Theorem. If $F$ satisfies (2), then $p \geqq 2 \pi / L$.

The fact that $p$ is bounded below by $c / L$ for some $c$ is surprising. This was first proved by Y. Sibuya (unpublished), who showed $p \geqq 2 / L$.

This estimate $2 \pi / L$ cannot be improved: If $n \geqq 2$ and

$$
x=\left(x_{1}, x_{2}, \cdots, x_{n}\right) \in R^{n},
$$

let $x_{1}^{\prime}=-L x_{2}, x_{2}^{\prime}=L x_{1}, x_{i}^{\prime}=0$ for $2<i \leqq n$, then (2) is satisfied letting $F(x)=\left(-L x_{2}, L x_{1}, 0, \cdots, 0\right)$, and all nonconstant solutions are periodic with period $2 \pi / L$.

To prove the theorem, we define the functions $f(t)=F(x(t))$ and $N(t)=\|f(t)\|$ and $y(t)=f(t) / N(t)$, for $t \in R$. The function $y(t)$ is a unit vector tangent to the periodic trajectory. The proof involves relating two facts about $(d / d t) y(t)$. Since (2) is satisfied, solutions of (1) are unique, so $N(t) \neq 0$ for any $t$. (If $N(t)=0$ for some $t$, we would have $N(t) \equiv 0$.) Since $x$ is a $C^{1}$ function, $x$ is Lipschitzean. Also, $f$ is a Lipschitz function of $t$ (with constant $L \sup _{t \in[0, p]}\left|x^{\prime}(t)\right|$ ). It is not hard to verify that $N$ and $y$ are also Lipschitzean, so in particular $f, N$ and $y$ are absolutely continuous, and therefore they are differentiable functions of $t$ almost everywhere. From now on, $t$ will always be assumed to be a point at which $f, N$, and $y$ have derivatives (which we denote $f^{\prime}, N^{\prime}$, and $\left.y^{\prime}\right)$. Note that $\|y\| \equiv 1$ so

$$
0=\frac{d}{d t}\|y(t)\|^{2}=2\left\langle y(t), y^{\prime}(t)\right\rangle
$$

Received by the editors November 7, 1968.

1 This work was supported in part by the National Science Foundation under grant GP-7846. 
where $\langle\cdot, \cdot\rangle$ is the inner product, i.e., $y$ and $y^{\prime}$ are perpendicular. Also $f^{\prime}=(y N)^{\prime}=y^{\prime} N+y N^{\prime}$. Since $y$ and $y^{\prime}$ are orthogonal for each $t$ and $N$ and $N^{\prime}$ are scalar functions, $\left\|f^{\prime}\right\|^{2}=\left\|y^{\prime} N\right\|^{2}+\left\|y N^{\prime}\right\|^{2}$, so

$$
\left\|f^{\prime}\right\| / N \geqq\left\|y^{\prime}\right\| \text {. }
$$

We can estimate $\left\|y^{\prime}\right\|$ as follows: for $s>0$

$$
\begin{aligned}
\|f(t+s)-f(t)\| & =\|F(x(t+s))-F(x(t))\| \leqq L\|x(t+s)-x(t)\| \\
& =L\left\|x^{\prime}(t)\right\| s+o(s)=L\|f(t)\| s+o(s),
\end{aligned}
$$

so

$$
\left\|f^{\prime}(t)\right\| \leqq L\|f(t)\|=L N(t)
$$

so

$$
\left\|y^{\prime}\right\| \leqq L \quad(\text { from (3) and (4)). }
$$

Variations of the next lemma were proved by Fenchel [1], Borsuk [3], and Milnor [2]. They let $x$ be $C^{2}$ with $\left\|x^{\prime}(t)\right\|=1$. Their proofs are substantially longer.

\section{LEMMA.}

$$
\int_{0}^{p}\left\|y^{\prime}\right\| d t \geqq 2 \pi
$$

The lemma says the variation of the unit vector in the direction $x^{\prime}$ in one period is at least $2 \pi$. This seems intuitively obvious and is clearly true in dimension $n=2$, but we delay the proof. (6):

The proof of the theorem is now completed by combining (5) and

$$
2 \pi \leqq \int_{0}^{p}\left\|y^{\prime}(t)\right\| d t \leqq \int_{0}^{p} L d t=L p,
$$

that is, $p \geqq 2 \pi / L$ and the theorem is proved.

We now only need to prove the lemma.

Proof of Lemma. Choose $t_{1}$ and $t_{1}+T$ in $[0, p], T>0$, such that $\left\|x\left(t_{1}+T\right)-x\left(t_{1}\right)\right\|=\sup _{\sigma, \tau \in[0, p]}\|x(\sigma)-x(\tau)\|$, (which is the "diameter" of the trajectory). Since

$$
x_{1}(t) \stackrel{\text { def }}{=} x\left(t-t_{1}\right)
$$

is also a periodic solution with period $p$, we may assume without loss that $t_{1}=0$. Write $v=x(0)-x(T)$. The function 


$$
u(t) \stackrel{\text { def }}{=} \frac{1}{2}\|x(t)-x(T)\|^{2}
$$

has a maximum at $t=0$ so

$$
0=\left.\frac{d}{d t} u(t)\right|_{t=0}=\left.\left\langle x(t)-x(T), x^{\prime}(t)\right\rangle\right|_{t=0}=\left\langle v, x^{\prime}(0)\right\rangle=\langle v, f(0)\rangle .
$$

Similarly, $\langle v, f(T)\rangle=\left\langle v, x^{\prime}(T)\right\rangle=0$, (letting $\left.u(t)=\frac{1}{2}\|x(t)-x(0)\|^{2}\right)$.

Claim. $\int_{0}^{T}\left\|y^{\prime}\right\| d t \geqq \pi$.

Let $S=\{x:\|x\|=1\}$. Then $y$ is a curve with values in $S$, and $\langle y(0), v\rangle=0$ and $\langle y(T), v\rangle=0$. The claim says that the length $\rho$ of the curve $y$ on $[0, T]$ is at least $\pi$.

Case 1. Assume $y(0)=-y(T)$. Then the shortest curve between $y(0)$ and $y(T)$ remaining on $S$ is a semicircle with radius 1 , so $\rho$ is at least $\pi$.

Case 2. Assume $y(0) \neq-y(T)$. Write $y^{0}=y(0), y^{T}=y(T), \gamma=y^{0}+y^{T}$, $m=y^{0}-y^{T}$. Then $\langle\gamma, m\rangle=\left\|y^{0}\right\|^{2}-\left\|y^{T}\right\|^{2}=0$. Let $a(t)=\langle y(t), \gamma\rangle /\langle\gamma, \gamma\rangle$. Then $a(T)=\langle(\gamma-m) / 2, \gamma\rangle /\langle\gamma, \gamma\rangle=1 / 2$. From the definition of $a(t)$, $y(t)-a(t) \gamma=h(t)$ for some function $h(t)$, where $\langle\gamma, h(t)\rangle=0$. Hence $a(t) \gamma+h(t)$ and $-a(t) \gamma+h(t)$ have the same norm $(=1)$. Recall $x^{\prime}=N y=N h+N a \gamma$ and $\int_{0}^{T} x^{\prime} d t=x(T)-x(0)=-m$ and $\langle m, \gamma\rangle=0$, so $\int_{0}^{T} N a=0$. Since $a(\cdot)$ is continuous there exists $T_{0} \in(0, T)$ such that $a\left(T_{0}\right)=0$. Define

$$
\begin{aligned}
y_{1}(t) & =y(t) & & \text { for } t \in\left[0, T_{0}\right] \\
& =h(t)-a(t) \gamma & & \text { for } t \in\left[T_{0}, T\right] .
\end{aligned}
$$

Then $y_{1}$ is continuous, and $y_{1}(t) \in S$ for all $t$ and the arc lengths for $y$ and $y_{1}$ on $[0, T]$ are the same. Note that $y_{1}(T)=h(T)-a(T) \gamma$ $=\left[y^{T}-\frac{1}{2} \gamma\right]-\frac{1}{2} \gamma=\left(\gamma-y^{0}\right)-\gamma=-y^{0}=-y_{1}(0)$. Therefore the shortest path from $y_{1}(0)$ to $y_{1}(T)$ in $S$ is half a great circle, which has the length $\pi$. Hence the length of $y_{1}$ (and $y$ ) is $\geqq \pi$, proving the claim.

The length of $y$ on $[T, p]$ (that is, of the other half of the trajectory) is also $\geqq \pi$ since this is the length that would be estimated if we had started at $x(T)$ instead of $x(0)$. Hence the length of $y$ on $[0, p]$ is at least $2 \pi$ and the lemma is proved.

REMARK. In $R^{3}, x(t)$ can be knotted. In this case Milnor [2] essentially proved when $F$ is $C^{1}$ that $\int_{0}^{p}\left\|y^{\prime}\right\|>4 \pi$. Applying this instead of the lemma, we get the theorem that $p>4 \pi / L$ when $x(t)$ is a knotted periodic solution.

Remark on the case $p=2 \pi / L$. If $x(t)$ is a periodic solution of (1) with period $p$ and if $p=2 \pi / L$, then (i) $\left\|x^{\prime}(t)\right\|$ is constant; (ii) the trajectory of $x$ is a circle, (with radius $\left\|x^{\prime}(t)\right\| / L$ and center $x(t)$ 
$\left.+x^{\prime \prime}(t) / L^{2}\right)$; and (iii) the two-dimensional disc $D$ whose boundary is the trajectory of $x$ is the union of concentric periodic orbits with period $p$ (with $F=0$ at the center), provided $F$ is defined on $D$.

Partial sketch of proof of remarks: if $p=2 \pi / L$, then where we had inequalities, we get the equalities

$$
\begin{gathered}
2 \pi=\int_{0}^{p}\left\|y^{\prime}\right\| d t, \quad \pi=\int_{0}^{T}\left\|y^{\prime}\right\| d t \\
\left\|y^{\prime}\right\|=\left\|f^{\prime}\right\| / N=L .
\end{gathered}
$$

From the first half of (8), since $\left\|f^{\prime}\right\|=\left\|y^{\prime} N\right\|^{2}+\left\|y N^{\prime}\right\|^{2}$, we get $\left.\left\|y N^{\prime}\right\|\right|^{2}$ $=0=N^{\prime}$, so $N$ is constant, and from (7), in the proof of the lemma we must have the length of the curve $y_{1}$ is $\pi$; hence $y_{1}$ (on $[0, T]$ and on $[T, p])$ describes half a great circle on $S$, and in fact one can see that $y$ describes half a great circle. Since $N \int_{0}^{p} y=\int_{0}^{p} x^{\prime}=0$, the path $y$ is a great circle on $[0, p]$. Therefore the trajectory of $x(t)$ is a planar "convex" curve. Since $N^{\prime}=0=\left\langle x^{\prime}, x^{\prime \prime}\right\rangle$, and $\left\|x^{\prime \prime}\right\|$ is constant (a.e.), the planar convex curve is a circle whose diameter is the (circumference) $/ \pi$, which $=p N / \pi$, i.e. $2 N / L$; hence $\|x(t)-x(t-p / 2)\|=2 N$ $/ L$. Since $f(t)=-f(t+p / 2)$,

$$
\|F(x(t))-F(x(t+p / 2))\|=2 N=L\|x(t)-x(t-p / 2)\| ;
$$

that is, the Lipschitz inequality in (2) is an equality for two points of the circle which are diametrically opposite. It follows that on the line between the points, i.e., points given by

$$
\begin{gathered}
z \stackrel{\text { def }}{=} s x(t)+[1-s] x(t-p / 2) \quad \text { for some } s \in[0,1], \\
F(z)=s F(x(t))+[1-s] F(x(t-p / 2)) .
\end{gathered}
$$

Any other choice of $F(z)$ would contradict (2). We now have an equation (on the disc $D$ ) which has concentric circles for solutions.

\section{REFERENCES}

1. W. Fenchel, Über Krümmung and Windung geschlossener Raumkurven, Math. Ann. 101 (1929), 238-252.

2. J. W. Milnor, On the total curvature of knots, Ann. of Math. (2) 52 (1950), 248257.

3. K. Borsuk, Sur la courbure totale des courbes fermés, Ann. Polon. Math. 20 (1947), 251-265.

\section{UNIVERSITY OF MARYLAND}

